This paper has already been published in Dyes and Pigments 69, 54-61 (2006).

\title{
Polymorph of dimethylacetamide-solvated crystals of 2,9-dichlorodithioketoquinacridone and their hydrogen bonding effect
}

\author{
Jin Mizuguchi, Tomonori Hoki and Takatoshi Senju \\ Department of Applied Physics, Graduate School of Engineering \\ Yokohama National University \\ Tokiwadai, Hodogaya, Yokohama, Japan
}

\begin{abstract}
2,9-Dichlorodithioketoquinacridone (DTQ-Cl) is a thionated derivative of industrially important 2,9-dichloroquinacridone used as colorants in painting and imaging industries. Crystallographic and electronic characterization has been carried out on single crystals of DTQ-Cl recrystallized from solution in dimethylacetamide (DMA). Two kinds of DMA-solvated single crystals were isolated in the form of DTQ-Cl/(DMA) 2 : modifications I and II. In both crystals, two DMA molecules are hydrogen-bonded to one DTQ-Cl molecule. Modifications I and II crystallize in different space groups: $P 21 / c$ and $P-1$, respectively. The DTQ-Cl molecules are arranged in a fashion "hunter's fence" in modification I; whereas all DTQ-Cl molecules are nearly on the molecular plane and stacked in a layered structure in modification II. MO calculations based on the X-ray coordinates revealed that the absorption bands for both DTQ-Cl and DTQ-Cl/(DMA) 2 are expected to appear at longer wavelengths in modification I than in modification II. However, the reverse is the case in experiment. This can be attributed to the difference in excitonic interactions along the stacking axis in modifications I and II.
\end{abstract}

Keywords: dithioquinacridone; crystal structure; electronic structure; polymorph 


\section{Introduction}

2,9-Dichlorodithioketoquinacridone (DTQ-Cl: Fig.1(a)) is a thionated derivative of industrially important 2,9-dichloroquinacridone used as colorants in painting and imaging areas [1]. We have so far investigated the crystal and electronic structure of a variety of quinacridone (QA) pigments: $\gamma$-QA [2], dithio-QA (i.e. DTQ; Fig.1(b)) [3,4], monomethyl-QA [5], dimethyl-QA [6] and 2,9-dimethyl-QA (2,9-DMQA) [7,8]. In these compounds, there are two types of molecular arrangement as characterized by $\mathrm{NH}^{\cdots} \mathrm{O}$ or $\mathrm{NH}^{\cdots} \mathrm{S}$ intermolecular hydrogen bonds. One type is that the QA molecules are arranged in a fashion "hunter's fence” to form a three-dimensional hydrogen bond network ( $\gamma$-QA \& DTQ). On the other hand, all molecules are nearly on the molecular plane in the form of a two-dimensional hydrogen bond network. In hydrogen-bonded QAs, a large bathochromic shift is commonly observed on going from solution to the solid state and is interpreted by excitonic interactions between transition dipoles arranged in a fashion "head-to-tail” [4,8].

Aside from colorant applications of QAs, thionated QAs are especially attractive because of an intense near-IR absorption used for laser printers and optical disks based on GaAsAl laser diodes [4]. DTQ, for example, exhibits an intense near-IR absorption [4]. Furthermore, thionated 2,9-dicholoro-QA (i.e. DTQ-Cl) seems to have more potential in view of the outstanding stability of 2,9-dichloro-QA. For this reason, we have initiated our investigation on the electronic characterization of DTQ-Cl on the basis of the crystal structure. Then, we obtained two kinds of dimethylacetamide (DMA)-solvated DTQ-Cl (modifications I and II) in which two DMA molecules are hydrogen-bonded to one DTQ-Cl molecule. In this connection, an attempt was made to clarify the crystal structure and characterize the polymorphic complexes with major focus on the intermolecular $\mathrm{NH} \cdots \mathrm{O}$ hydrogen bonds.

Our previous study on the crystal and electronic structure of DTQ is summarized as follows. Fig.2 shows the crystal structure of DTQ [3]. There are intermolecular hydrogen bonds between the $\mathrm{NH}$ group of one DTQ molecule and the $\mathrm{S}$ atom of the neighboring one. One molecule is hydrogen-bonded to four neighboring molecules. Thus, the molecules are arranged in a fashion "hunter's fence”. In the solid state, there are three characteristic absorption spectra in evaporated films as shown in Fig.3 [(a) as evaporated, (b) phase I and (c) phase II]. Among these, only spectrum (c) is near-IR-active [4]. The extent of the bathochromic shift upon crystallization is about $72 \mathrm{~nm}\left(\mathrm{ca} 1550 \mathrm{~cm}^{-1}\right)$ in phase I and $108 \mathrm{~nm}$ 
(ca $2200 \mathrm{~cm}^{-1}$ ) in phase II.

\section{Experiment}

\subsection{Preparation of DTQ-Cl and its solvated single crystals}

DTQ-Cl was prepared by thionation of commercially available 2,9-dichloroquinacridone with a Lawesson's regent [9]. Single crystals of DTQ-Cl were grown by recrystallization from solution in dimethylacetamide (DMA). Two kinds of DMA-solvated single crystals (modifications I and II) were isolated. On the other hand, no solvent-free crystals were obtained. Single crystals of modification I were grown in the form of needles while platelet in modification II.

The crystal growth from the vapor phase failed because of the decomposition of DTQ-Cl around $350{ }^{\circ} \mathrm{C}$. Dimethylformamide-solvated crystals were also obtained. This structural result is given in Appendix.

\subsection{Structure analysis}

X-ray diffraction data were collected at $93 \mathrm{~K}$ on a R-AXIS RAPID-F diffractometer from Rigaku using $\mathrm{CuK \alpha}$ radiation ( $\lambda=1.5418 \AA$ ). The structure was solved by direct methods using Shelxs-97 and refined by full-matrix least squares on $F^{2}$ using Shelxl-97 [10].

\subsection{Molecular orbital (MO) calculations}

MO calculations were made using Quantum CAChe Ver.3.2 from Fujitsu, Ltd. The geometry was first optimized for DTQ-Cl and DTQ-Cl/(DMA)2, using the AM1 Hamiltonian. Then, the absorption bands were calculated for the optimized DTQ-Cl using the INDO/S Hamiltonian. The same spectroscopic calculations were also carried out on two kinds of DMA-solvated complexes extracted from the crystal lattice.

\subsection{Equipment}


UV-vis spectra in solution were recorded on a Shimadzu UV-2400PC spectrophotometer. Polarized reflection spectra were measured on single crystals by means of a microscope-spectrophotometer (UMSP 80 from Carl Zeiss) equipped with an R928 photomultiplier (HTV). An epiplan pol objective $(\times 8)$ was used together with a Nicol-type polarizer. Reflectivities were corrected relative to the reflection standard of silicon carbide.

\section{Results and Discussion}

\subsection{Crystal structure of modifications I and II}

Table 1 details the crystallographic parameters for DMA-solvated complexes: modifications I and II. In both crystals, two DMA molecules are hydrogen-bonded to one DTQ-Cl molecule. The molecular symmetry is $C_{1}$ in modification I while $C_{\mathrm{i}}$ in modification II. The complexes are arranged in a different way as characterized by space groups $P 21 / c$ and $P-1$, respectively.

Fig.4 shows the ORTEP plot of DTQ-Cl/(DMA) $)_{2}$ in modification I as characterized by $C_{1}$ symmetry. The solvent molecules are nearly on the molecular plane of DTQ-Cl. On the other hand, the DMA molecule in DTQ-Cl/(DMA) $)_{2}$ in modification II is disordered with a site occupancy of 0.5 (Figs.5(a) and Fig.5(b)). In both complexes, there are $\mathrm{NH} \cdots \mathrm{O}$ intermolecular hydrogen bonds between the $\mathrm{NH}$ group of DTQ-Cl and the $\mathrm{O}$ atom of DMA. The N/O distance and the NH/O angle are $1.894 \AA$ and $176^{\circ}$ in modification I, respectively: whereas the N/O distance and the NH/O angle are $1.868 \AA$ and $173^{\circ}$ in modification II, respectively. These hydrogen bonds are considered to be relatively strong, since the N/O distance is short and the $\mathrm{NH} / \mathrm{O}$ angle is close to $180^{\circ}$ in both crystals.

Figs.6(a) and 6(b) show the projection of the crystal structure of modification I onto the $(a, c)$ plane as well as the molecular stack along the $b$-axis. All DMA molecules are almost on the molecular plane of DTQ-Cl. Figs.7(a) and 7(b) show the molecular arrangement on the $(b, c)$ plane and along the molecular stack in modification II, respectively. It is apparent in both crystals that there are columns composed of DTQ-Cl molecules as well as of DMA molecules along the $b$ - and $a$-axes in modifications I and II, respectively. Thus, the DTQ-Cl molecules work as the spacer to separate DTQ-Cl molecules. In other words, the DMA molecule blocks the formation of $\mathrm{NH}^{\cdots} \mathrm{S}$ intermolecular hydrogen bonds between two 
DTQ-Cl molecules.

In modification I (P21/c) as shown in Fig.6(b), the DTQ-Cl molecules are arranged in such a way to cross each other at angles of about $60^{\circ}$ ("hunter's fence”). The present arrangement is similar to that of DTQ as shown in Fig.2. On the other hand, in modification II (P-1), the DTQ-Cl molecules are almost on the molecular plane and the DMA molecules are located almost perpendicular to the molecular plane of DTQ-Cl (Fig.7(b)). This layered arrangement DTQ-Cl is similar to that of 2,9-DMQA [7]. Furthermore, it is of great interest to note that the absorption maxima of modifications I and II are slightly different as discussed below.

\subsection{Solution spectra and the effect of $\mathrm{H}$-bond on the spectral shift}

Fig.8 shows the solution spectra of DTQ-Cl measured in 1,4-dioxane, DMA and DMSO (dimethylsulfoxide). In all spectra, there observed a progression of absorption bands starting from $640 \mathrm{~nm}$ (for example, in DMA) toward shorter wavelengths. Since the absorption edge of the longest-wavelength band is quite steep and the subsequent bands are equally spaced (about $1400 \mathrm{~cm}^{-1}$ ), the longest-wavelength band is assigned to the $0-0$ transition and the second longest wavelength band to the $0-1$ transition and so on. This assignment is also borne out by MO calculations as described below.

It is also to be noted that the absorption maximum is slightly displaced toward longer wavelengths in sequence of 1,4-dioxane, DMA and DMSO. This is the increasing order of the polarity of the solvent. The present bathochromic shift is closely linked with the hydrogen bond formation between the NH group of DTQ-Cl and the $\mathrm{O}$ atom of solvents as supported by

${ }^{1} \mathrm{H}-\mathrm{NMR}$ experiments (not shown here). The proton signal is shifted toward up-field as the solution temperature is increased from RT to $100{ }^{\circ} \mathrm{C}$. At the same time, the extent of the bathochromic shift is significantly reduced due to the weakening of the hydrogen bond caused by enhanced kinetic motion of molecules at high temperatures.

\subsection{MO calculations}

Table 2 details the results of the spectroscopic calculations for DTQ-Cl and DTQ-Cl/(DMA $)_{2}$ in modifications I and II together with the experimental values in solution and in the solid state. It is of interest to note that the intermolecular hydrogen bond between 
the NH group of DTQ-Cl and the O atom of DMA brings about a slight bathochromic shift in the optimized geometry as well as in modifications I and II. In addition, the absorption maxima calculated for both DTQ-Cl and DTQ-Cl/(DMA) $)_{2}$ for the optimized geometry are in good agreement with those for modification II: 426 and $435 \mathrm{~nm}$, respectively. This suggests that the molecular conformation optimized for DTQ-Cl and DTQ-Cl/(DMA) 2 is assumed quite similar to that of modification II. On the other hand, the absorption maxima are supposed to appear at longer wavelengths in modification I (440 and $448 \mathrm{~nm}$, respectively) than in modification II. However, in experiment, the opposite is the case as presented below.

\subsection{Polarized reflection spectra measured on single crystals}

Fig.9 shows the polarized reflection spectra measured on the $(a, c)$ plane of modification I. The projection onto the $(a, c)$ plane is given in Fig.6(a). A prominent reflection band appears around $690 \mathrm{~nm}$, accompanied by two small bands around 632 and $586 \mathrm{~nm}$ for polarization parallel to the transition dipole as deduced from MO calculations. On the other hand, these reflection bands are completely quenched for polarization perpendicular to the direction of the transition dipole. This result indicates that the direction of the transition dipole, as determined experimentally here, is in agreement with the calculated one. The same spectroscopic behavior is also observed for modification II as shown in Fig.10. However, the reflection maximum appears around $700 \mathrm{~nm}$. This band is present at longer wavelengths by about $10 \mathrm{~nm}$ (ca $210 \mathrm{~cm}^{-1}$ ) as compared with that of modification II. The above results indicate that the bathochromic shift, upon crystallization relative to the absorption maximum in a DMA solution (Fig.8), amounts to $27.5 \mathrm{~nm}\left(\mathrm{ca} 602 \mathrm{~cm}^{-1}\right)$ and $37.5 \mathrm{~nm}\left(\mathrm{ca} 808 \mathrm{~cm}^{-1}\right.$ ) for modifications I and II, respectively.

(There is another thing to be noted in the polarized spectra (Fig.9) that a gradual increase in reflectivity is recognized in the region from about 720 to $760 \mathrm{~nm}$, followed by a constant reflectivity up to $800 \mathrm{~nm}$ for polarization perpendicular to the transition dipole. This is due to the reflection from the rear plane of the single crystal. This often occurs in general in the spectral region where no absorption band exists. This also exerts an influence in the spectrum above $720 \mathrm{~nm}$ for polarization parallel to the transition dipole.)

\subsection{Bathochromic shift due to $\mathrm{NH} \cdots \mathrm{O}$ hydrogen bonds in DTQ-Cl/(DMA $)_{2}$}


As stated in section 3.2, the absorption band in solution is red-shifted when the $\mathrm{NH} \cdots \mathrm{O}$ intermolecular hydrogen bonds are formed between the $\mathrm{NH}$ group of $\mathrm{DTQ}-\mathrm{Cl}$ and the $\mathrm{O}$ atom of solvents. Before we go into details on the hydrogen bond, it is important to bear in mind that the electronic system of DTQ-Cl is composed of the five condensed-rings as the chromophore and the two pairs of $\mathrm{NH}$ (donor) and $\mathrm{C}=\mathrm{O}$ (acceptor) groups as the auxochrome. The present push-pull system facilitates the electron transfer, upon light absorption, from the donor to the acceptor part across the chromophore. The direction of the electron transfer is actually the direction of the transition dipole as will be discussed in the next section. Then, if the electro-donating power is enhanced due to the formation of $\mathrm{NH}^{\cdots} \mathrm{O}$ hydrogen bonding, this may lead to a bathochromic shift. In addition to its primarily electrostatic character, the hydrogen bond has some covalent nature correspond to the scheme: $\mathrm{N}^{-}-\mathrm{H}^{+} \cdots \mathrm{O}=\mathrm{C} \Leftrightarrow \mathrm{N}^{-}-\mathrm{H}-$ $\mathrm{O}^{+}=\mathrm{C}$. This second charge transfer form involves electron donation from the oxygen into the $\mathrm{N}-\mathrm{H}$ bond increasing the electron density on the nitrogen atom. Then, this increased electron density (i.e. enhanced electro-donating power) facilitates the electron transfer to the acceptor part, contributing to the bathochromic shift. Furthermore, if the polarity of the solvent is increased, more negative charge will appear on the $\mathrm{N}$ atom to enhance a bathochromic shift. This explains why the bathochromic shift becomes significant in sequence of the increasing order of the polarity of solvents.

\subsection{Difference in absorption maxima in modifications I and II}

In MO calculations described in section 3.3, the absorption bands based on the X-ray coordinates for $\mathrm{DTQ}-\mathrm{Cl} /(\mathrm{DMA})_{2}$ are expected to appear at longer wavelengths in modification I (448 nm) than in modification II (435 nm) (Table 2). However, the reverse is the case in polarization experiments measured on single crystals (Figs.9 and 10): 690 and 700 $\mathrm{nm}$ for modifications I and II, respectively. The difference in wavelength amounts to $10 \mathrm{~nm}$ (about $210 \mathrm{~cm}^{-1}$ ). This is obviously attributed to intermolecular interactions in the solid state.

In pigment systems where the molecular absorption coefficient (i.e. proportional to the square of transition dipole) is quite large, the interaction between transition dipoles is known to play the major role in intermolecular interactions in the solid state $[11,12]$. The interaction energy $\left(\Delta E_{\text {exciton }}\right)$ is given by the dipole-dipole equation [13]: $\Delta E_{\text {exciton }}=|\mu|^{2}\left(1-3 \cos ^{2} \theta\right) / r^{3}$, 
where the transition dipole is denoted by $\mu$, the distance and angle between two transition dipoles by $r$ and $\theta$, respectively. As evident from the present equation, the overall shift energy is determined by the strength of the interneighbor coupling $\left(|\mu|^{2}\right)$ which directly depends on the absorption coefficient of the molecule as well as on the mutual relative orientation of the transition dipoles in molecular assemblies. That is, the term $\left(1-3 \cos ^{2} \theta\right) / r^{3}$ determines the geometrical relationship of transition dipoles correlated with the crystal structure. Since this term falls off as the inverse cube of distance, most of the interaction would come from the nearest neighbors.

In fact, in DTQ, large bathochromic shifts of about $1550 \mathrm{~cm}^{-1}$ and $2200 \mathrm{~cm}^{-1}$ appear upon

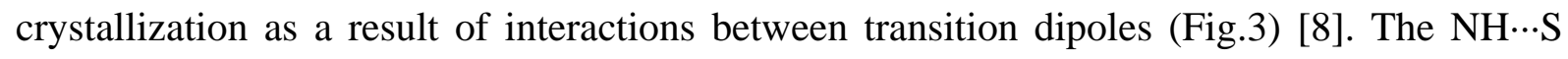
intermolecular hydrogen bond aligns the transition dipoles in a fashion "head-to-tail”, leading to the bathochromic shift. However, in DMA-solvated DTQ-Cl systems, the DMA molecules are hydrogen-bonded to DTQ-Cl molecules to block the formation of $\mathrm{NH} \cdots \mathrm{S}$ hydrogen bonds between DTQ-Cl molecules. Therefore, the excitonic interactions between transition dipoles are considered to be quite small on the molecular plane. However, a small effect due to excitonic interactions can still appear along the stacking axis (Figs.6 \& 7) since the absorption coefficient of DTQ-Cl is quite large (Fig.8). Our calculations using the dipole-dipole equation on the basis of the X-ray coordinates indicate that the bathochromic shift is slightly larger in modification II than in modification I by a few hundred wavenumbers. This is consistent with the tendency of the experimental result.

\section{Conclusions}

Crystallographic and electronic characterization has been carried out on the modifications I and II of DTQ/(DMA)2. The conclusions can be summarized as follows.

1. Modifications I and II crystallize in two different space groups: $P 21 / C$ and $P-1$, respectively. In both crystals, two DMA molecules are hydrogen-bonded to one DTQ-Cl molecule. The DTQ-Cl molecules are arranged in a fashion "hunter's fence" in modification I; whereas all DTQ-Cl molecules are nearly on the molecular plane and stacked in a layered structure along the $a$-axis in modification II.

2. The formation of the $\mathrm{NH} \cdots \mathrm{O}$ intermolecular hydrogen bonds between the $\mathrm{NH}$ group of DTQ-Cl and the O atom of DMA in solution bring about a slight bathochromic shift. This 
is due to the enhanced electron density on the $\mathrm{N}$ atom that facilitates the electron transfer from the donor $(\mathrm{NH})$ to the acceptor $(\mathrm{C}=\mathrm{O})$.

3. The absorption bands for both DTQ-Cl and DTQ-Cl/(DMA) 2 are expected to appear at longer wavelengths in modification I than in modification II according to MO calculations based on the X-ray coordinates. However, the reverse is the case in experiment. This can be attributed to the difference in excitonic interactions along the stacking axis in modifications I and II. 


\section{References}

[1] Herbst M, Hunger K. Industrial Organic Pigments. Weinheim: VCH; 1997.

[2] Mizuguchi J; Sasaki, T, Tojo, K. Z. Kristallogr. NCS, 2002; 217: 249.

[3] Mizuguchi J; Rihs, G. Acta Crystallogr. 1992; C48:1 553.

[4] Mizuguchi, J; Rochat, A C; Rihs, G. Ber. Bunzenges. Phys. Chem., 1994; 98, 19.

[5] Mizuguchi, J; Senju, T. Z. Kristallogr. NCS, 2002; 217: 523.

[6] Mizuguchi J; Senju, T. Acta Crystallogr. 2003; E59:0232.

[7] Mizuguchi, J; Senju, T; Sakai, M. Z. Kristallogr. NCS, 2002; 217: 525.

[8] Mizuguchi, J; Senju, T. Proc. of NIP 19, 2003: 678.

[9] Rochat, A C; Jaffe, E E; Mizuguchi, J. Eur. Pat. Appl. EP267873, 1988.

[10] Sheldrick, G M. Shelxs-97 and Shelxl-97, The University of Göttingen, Germany, 1997.

[11] Mizuguchi, J. J. Phys. Chem. A, 2000;104:1817.

[12] Mizuguchi, J; Tojo, K. J. Phys. Chem. A, 2002; 106: 767.

[13] Kasha, M. Spectroscopy of the Excited State, B. Di Bartolo Ed., NATO Advanced Study Institute Series: Series B, Vol. 12, Plenum Press, NY, 1976; 337. 
Figure captions

Fig.1 Molecular structure: (a) DTQ-Cl and (b) DTQ.

Fig.2 Molecular arrangement of DTQ (phase I). The transition dipole is designated by dotted lines. $P 21 / c, a=10.657(1), b=5.120(1), c=14.813(2) \AA, \beta=109.06(1)^{\circ}$.

Fig.3 Absorption spectra of evaporated DTQ: (a) as evaporated, (b) vapor-treated for 5 min (phase I) and (c) vapor-treated for 15 min (phase II).

Fig.4 ORTEP plot of DTQ-Cl for modification I. DMA is nearly on the molecular plane of DTQ-Cl.

Fig.5 ORTEP plots of DTQ-Cl for modification II where the DMA molecule is disordered: (a) DMA is nearly perpendicular to the molecular plane of DTQ-Cl and (b) DMA molecule is nearly on the molecular plane of DTQ-Cl. The site occupancy of the disordered atoms is 0.5 .

Fig.6 Molecular arrangement of modification I: (a) projection onto the $(a, c)$ plane and (b) molecular stack along the $b$-axis. The direction of the transition dipole $(\mu)$ as deduced from MO calculations is designated by dotted lines.

Fig.7 Molecular arrangement of modification II: (a) projection onto the $(b, c)$ plane and (b) molecular stack along the $a$-axis. The DMA conformation shown in Fig.5(a) is used for the present illustration. The direction of the transition dipole $(\mu)$ as deduced from MO calculations is designated by dotted lines.

Fig.8 Solution spectra in three different solvents: 1,4-dioxane, DMA and DMSO. All spectra are adjusted to the absorption maximum in DMSO.

Fig.9 Polarized reflection spectra measured on the $(a, c)$ plane of modification I. The projection onto the $(a, c)$ is given in Fig.6(a) which also includes the direction of the transition dipole $(\mu)$.

Fig.10 Polarized reflection spectra measured on the $(b, c)$ plane of modification II. The projection onto the $(b, c)$ plane is shown in Fig. 7(a) which also includes the direction of the transition dipole $(\mu)$.

Fig.11 ORTEP plot of DTQ-Cl/(DMF) 2 .

Fig.12 Molecular arrangement of DTQ-Cl/(DMF) $)_{2}$ : (a) projection onto the $(b, c)$ plane and (b) along the molecular stack. 


\section{Appendix}

\section{Crystal structure of DTQ-Cl/(DMF)}

Single crystals of $\mathrm{DTQ}-\mathrm{Cl} /(\mathrm{DMF})_{2}$ were grown by recrystallization from solution in dimethylformamide. The crystallographic parameters are: monoclinic, $C 2 / c, Z=4, a=$ 33.254(4) $\AA, b=4.2677(6) \AA, c=21.586(3) \AA, \beta=123.876(9)^{\circ}$. The ORTEP plot as well as the molecular arrangement is shown in Figs.11 and 12, respectively. 

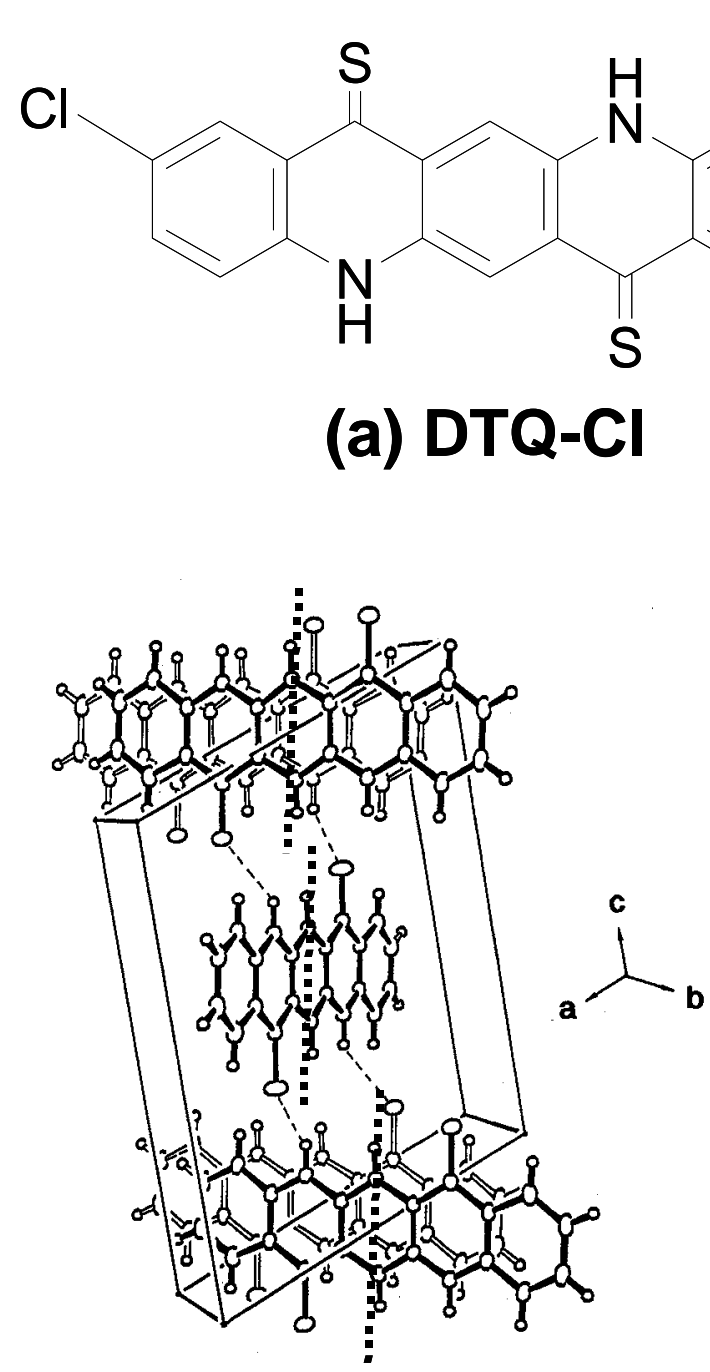

....-transition dipole

Fig. 2

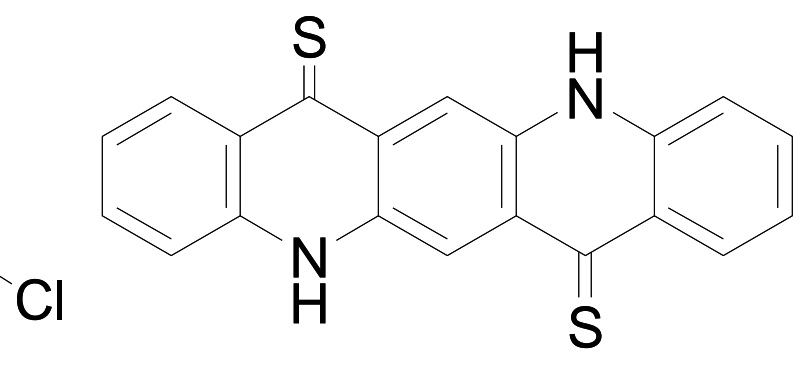

(b) DTQ

Fig. 1

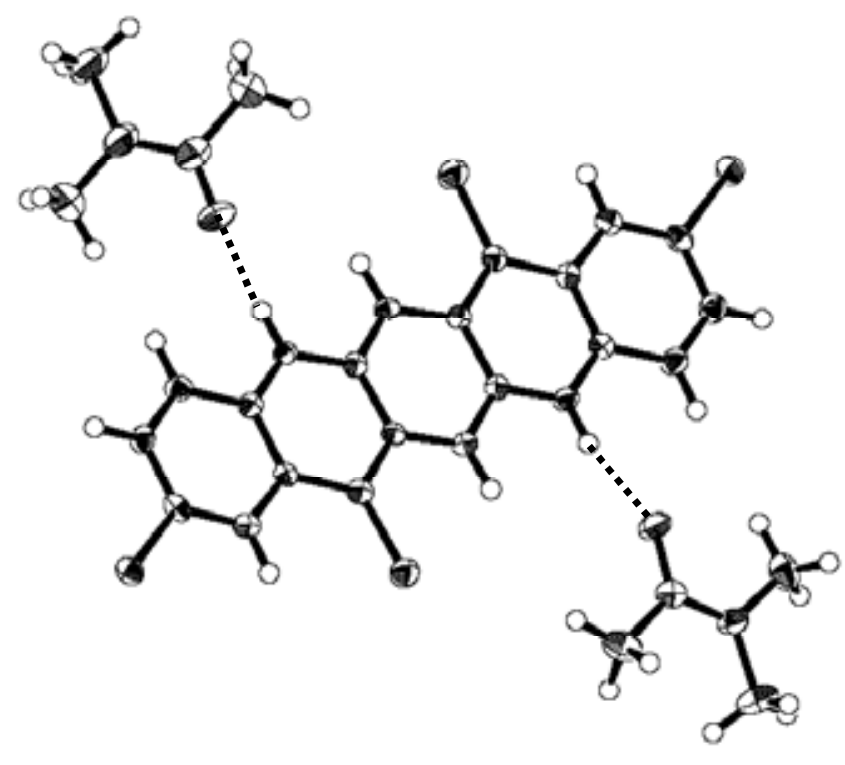

..... hydrogen bond

Fig. 4

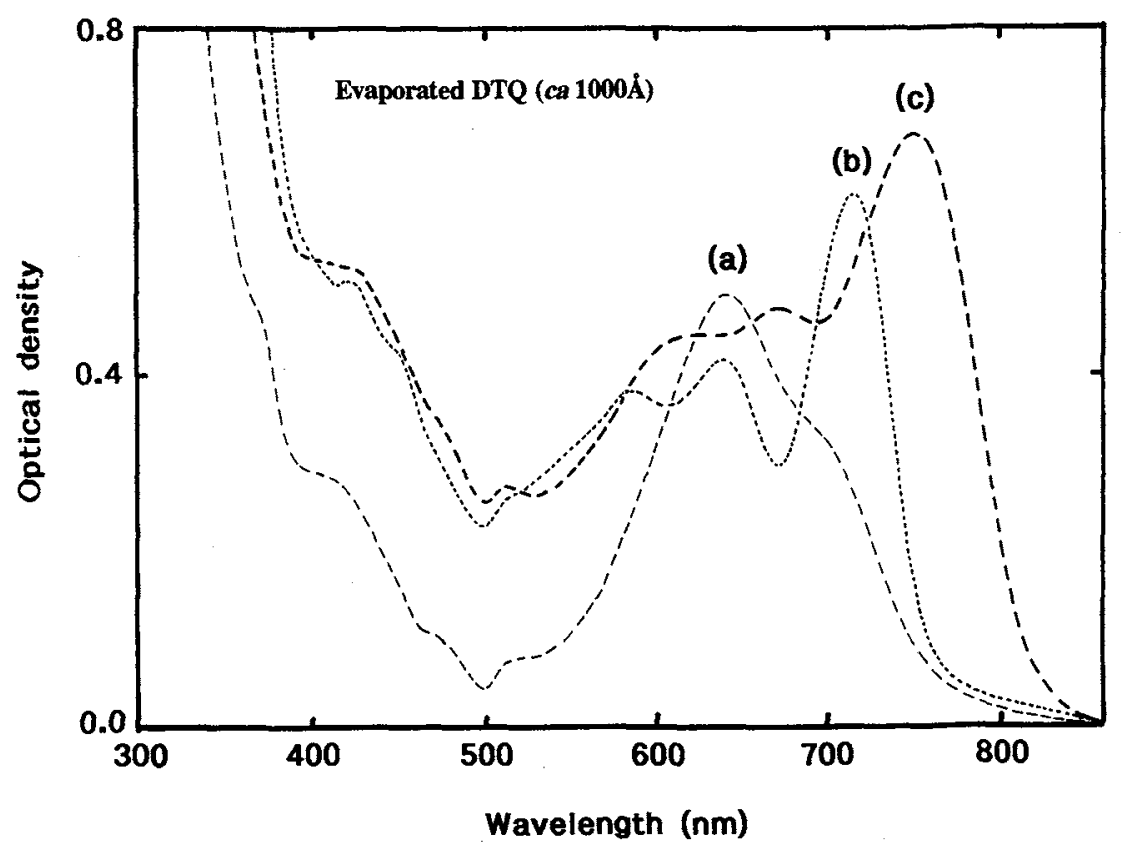

Fig. 3 
(a)

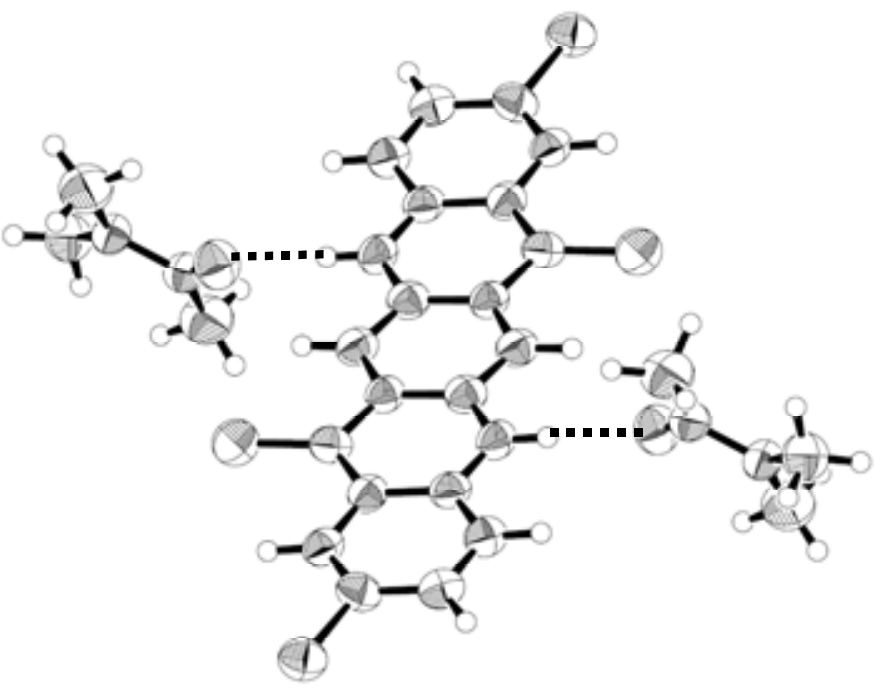

(b)

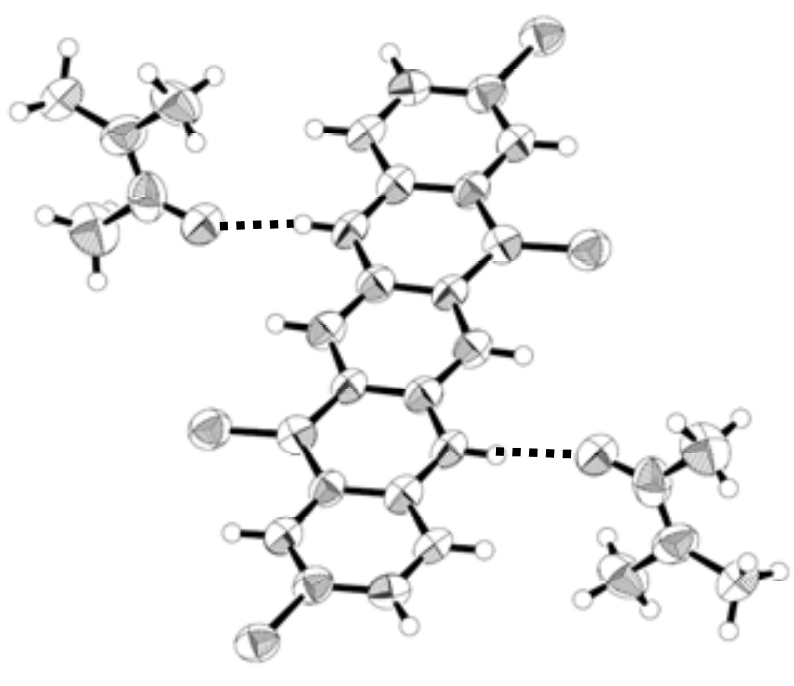

..... hydrogen bond

Fig. 5

(a)

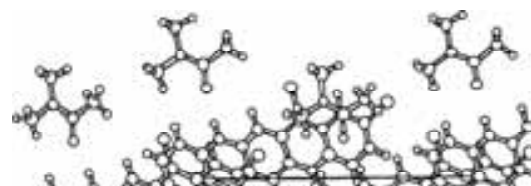

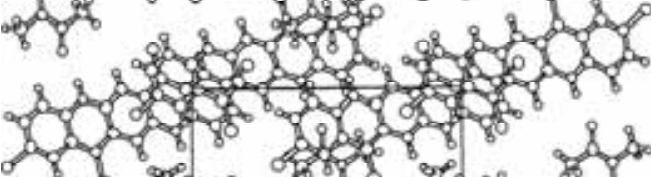
4 ress os os of Af of Ab

..... transition dipole (b)
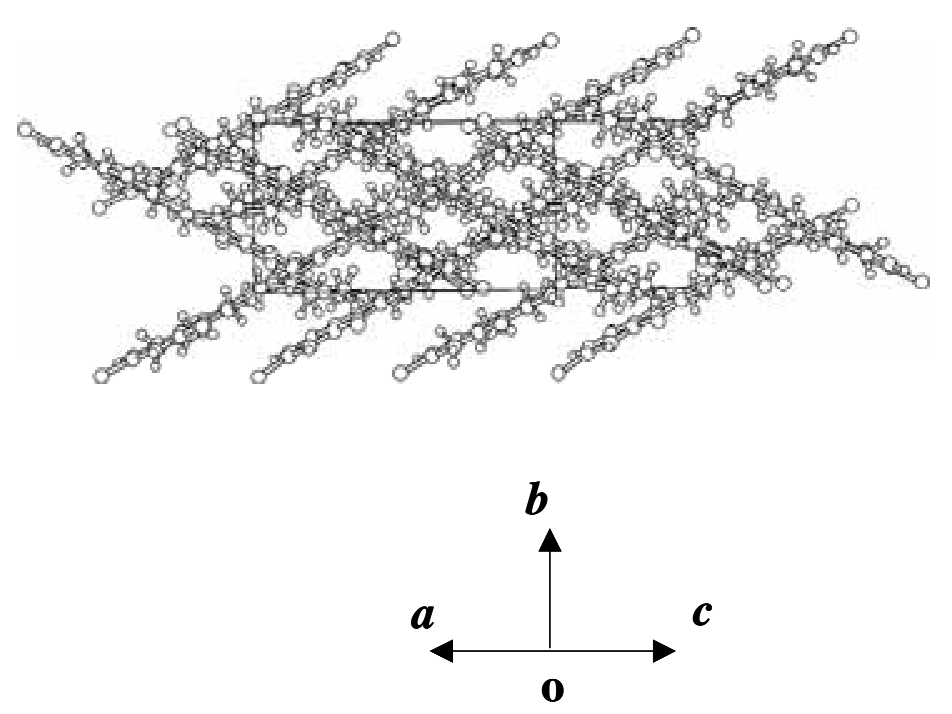

Fig. 6 
(a)

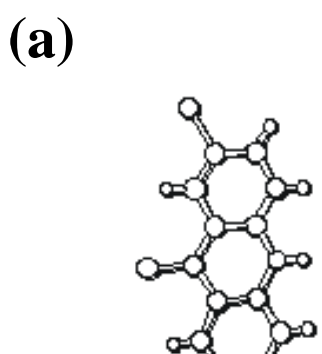<smiles>O=C1C(O)C2CC3CC1C3C2</smiles><smiles>C1CCOCC1</smiles><smiles>O=C1C=CCCC1=O</smiles><smiles></smiles>

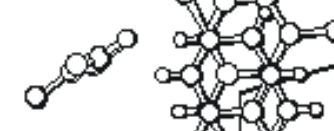

os of $=09=0$
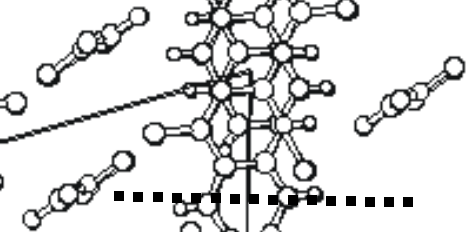

$=0=$

of

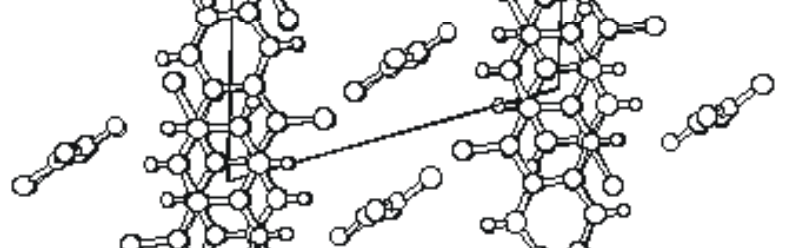

$$
0
$$

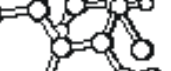

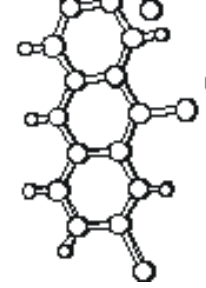

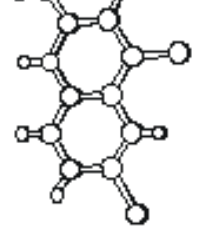

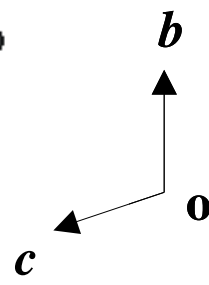

transition dipole

Fig. 7

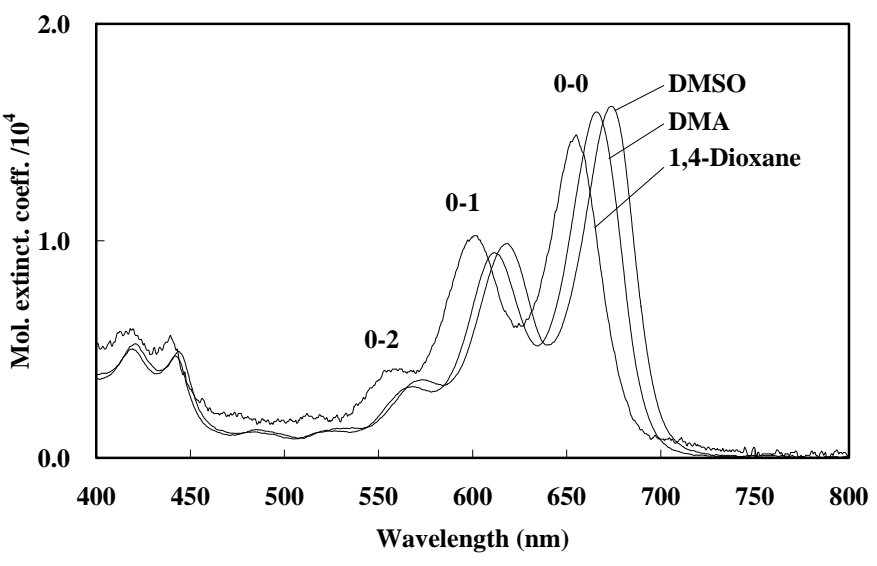

Fig. 8 (b)
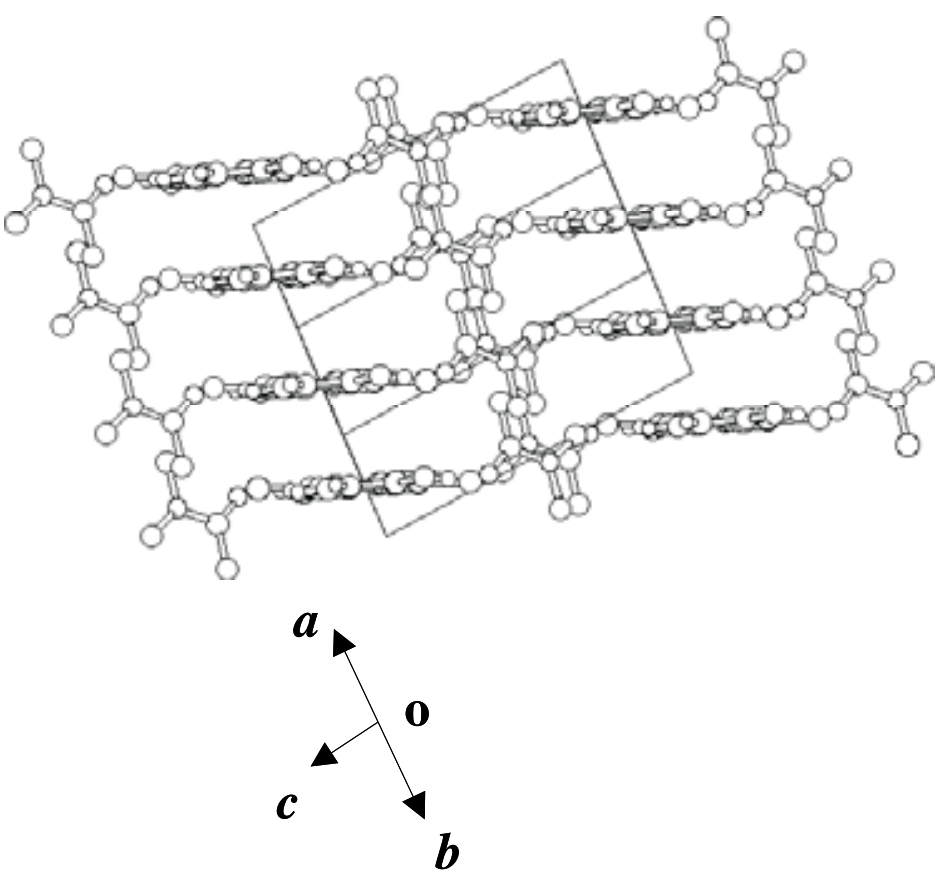

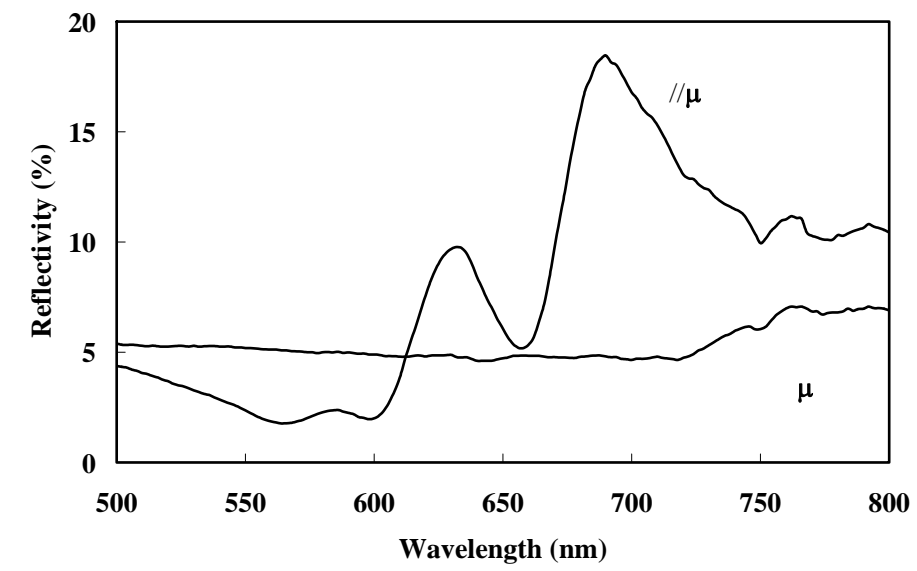

Fig. 9 


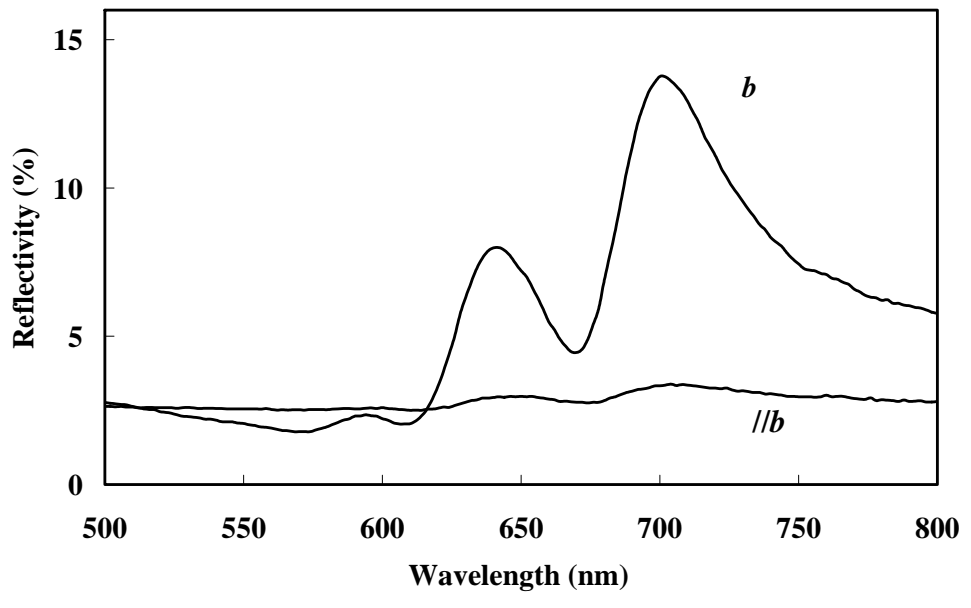

Fig. 10

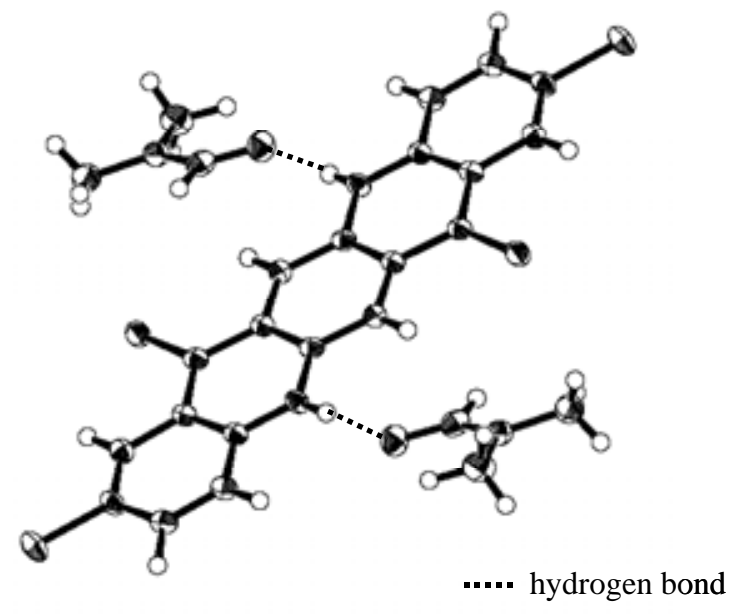

Fig. 11 (a)

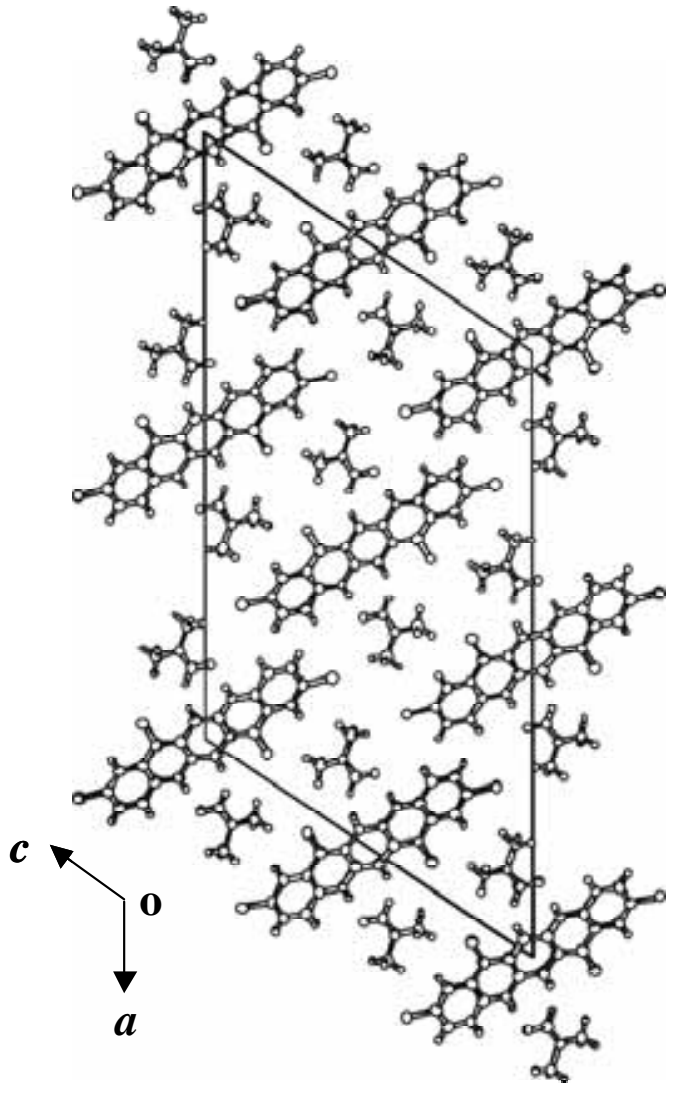

(b)

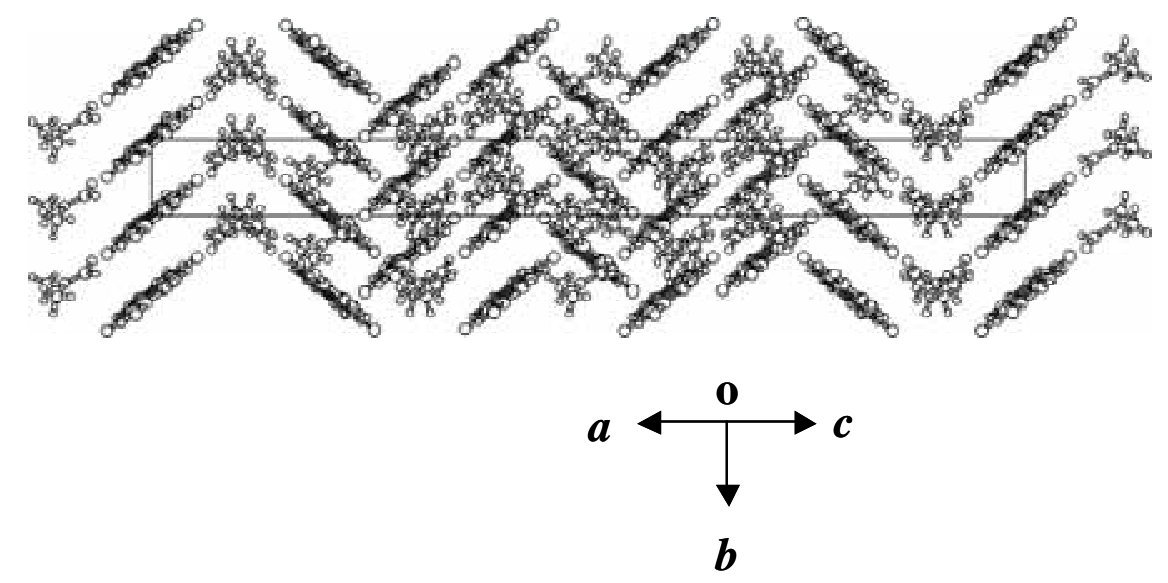

Fig. 12 